This is the author's final, peer-reviewed manuscript as accepted for publication. The publisher-formatted version may be available through the publisher's web site or your institution's library.

\title{
A counterexample to a uniqueness result
}

A. G. Ramm

\section{How to cite this manuscript}

If you make reference to this version of the manuscript, use the following information:

Ramm, A. G. (2002). A counterexample to a uniqueness result. Retrieved from http://krex.ksu.edu

\section{Published Version Information}

Citation: Ramm, A. G. (2002). A counterexample to a uniqueness result. Applicable Analysis, 81(4), 833-836.

Copyright: ( 2002 Taylor \& Francis Ltd

Digital Object Identifier (DOI): doi:10.1080/0003681021000004456

\section{Publisher's Link:}

http://www.tandfonline.com/doi/abs/10.1080/0003681021000004456\#.UYwATUr7BoE

This item was retrieved from the K-State Research Exchange (K-REx), the institutional repository of Kansas State University. K-REx is available at http://krex.ksu.edu 
A.G.Ramm, A counterexample to a uniqueness result of Cox and Thompson, Applic. Analysis, 81, N4, (2002), 833-836. 


\title{
A counterexample to a uniqueness result
}

\author{
A.G. Ramm *† \\ Mathematics Department, Kansas State University, \\ Manhattan, KS 66506-2602, USA \\ ramm@math.ksu.edu
}

\begin{abstract}
A counterexample is given to the uniqueness result given in the paper by J.Cox and K.Thompson, "Note on the uniqueness of the solution of an equation of interest in inverse scattering problem", J. Math.Phys., 11, N3, (1970), 815-817.
\end{abstract}

\section{Introduction}

In [3] the authors claimed that the integral equation

$$
h(s)=-\int_{0}^{r} g(s, t) h(t) t^{-2} d t
$$

where $h(0)=0$, has only the trivial solution for all $r>0$. Here

$$
g(s, t)=\sum_{m \in S} \gamma_{m} u_{m}(t) v_{m}(s), \quad s>t
$$

the set $S$ is a finite set of distinct real numbers from the interval $(-0.5, \infty), u_{m}$ and $v_{m}$ are the regular and irregular Bessel-Riccati functions, defined e.g. in $[2], g(s, t)$ is symmetric, and $\gamma_{m}$ satisfy the following equation:

$$
\sum_{m \in S} \gamma_{m}[m(m+1)-l(l+1)]^{-1}=1, \quad l \in T,
$$

where $T$ is a finite set of distinct real numbers from the interval $(-0.5, \infty)$, and the sets $T$ and $S$ are disjoint. This uniqueness result is crucial for the arguments in [4]. For references on the inverse scattering with fixed-energy data see [1]-[9]. In this note a counterexample to the uniqueness claim from [3] is constructed. This counterexample invalidates the arguments in [3]. The uniqueness of the solution of similar equations in [2] (see equations (12.1.2) and (12.2.1) on pp.195-196 in [2]) and [5] does not hold for some $r>0$, in general, also, as follows from the results in [10] and [8].

${ }^{*}$ Math subject classification: 34R30; PACS: 03.80.+r. 03.65.Nk

†This paper was written when the author was visiting Institute for Theoretical Physics, University of Giessen. The author thanks DAAD for support and Professor W.Scheid for discussions 


\section{A counterexample}

Take the single-element sets $S=\{0\}$ and $T=\{2\}$. In this case equation (1.2) yields $\gamma_{0}=-6, g(s, t)=-6 u_{0}(t) v_{0}(s):=-6 g_{0}(s, t)$ for $t<s$ and $g_{0}=u_{0}(s) v_{0}(t)$ for $t>s$. Note that $\left(d^{2}+1\right) g_{0}=\delta(s-t)$, where $\delta(s-t)$ is the delta-function, and $d^{2}$ stands for the second derivative. Therefore, applying the operator $d^{2}+1$ to equation (1.1), one gets $\left(d^{2}+1\right) h=6 s^{-2} h$. This equation has a nontrivial, regular at zero, solution $c u_{2}(s)$, where $c \neq 0$ is a constant. Without loss of generality we take $c=1$ in what follows.

Note that $u_{0}(r)=\sin r, v_{0}(r)=-\cos r, u_{2}(r)=\left(3 r^{-2}-1\right) \sin r-3 r^{-1} \cos r$, and the Wronskian $u_{0} v_{0}^{\prime}-u_{0}^{\prime} v_{0}=1$.

Define the function

$$
p(r):=v_{0}(r) u_{2}^{\prime}(r)-v_{0}^{\prime}(r) u_{2}(r) .
$$

One may check, using the explicit formulas for $v_{0}$ and $u_{2}$, that

$$
p(r)=1-\frac{3+3 \cos ^{2}(r)}{r^{2}}+\frac{3 \sin (2 r)}{r^{3}} .
$$

Using this explicit formula, one checks that $p(r)=-\frac{r^{2}}{5}+o\left(r^{2}\right)$ as $r \rightarrow 0$, and that $p(r)>0$ as $r \rightarrow \infty$. In fact, $p(r) \rightarrow 1$ as $r \rightarrow \infty$. Since $p(r)$ is continuous on $(0, \infty)$, one concludes that:

There is a number $R>0$ such that $p(R)=0$.

Let us now prove the following:

Claim: Equation (1.1) has a nontrivial solution $u_{2}(s)$ if $r=R$.

Proof of the claim:

One has:

$$
-\int_{0}^{r} g(s, t) u_{2}(t) t^{-2} d t=\int_{0}^{r} g_{0}(s, t) 6 u_{2}(t) t^{-2} d t=\int_{0}^{r} g_{0}(s, t)\left(d^{2}+1\right) u_{2}(t) d t:=J .
$$

Integrating by parts twice and taking into account that $u_{0}$ and $u_{2}$ vanish at the origin, one gets:

$$
J=u_{2}(s)\left[u_{0}(s) v_{0}^{\prime}(s)-u_{0}^{\prime}(s) v_{0}(s)\right]+u_{0}(s)\left[v_{0}(r) u_{2}^{\prime}(r)-v_{0}^{\prime}(r) u_{2}(r)\right]=u_{2}(s)+p(r) u_{0}(s) .
$$

We have used above the formula for the Wronskian: $u_{0} v_{0}^{\prime}-u_{0}^{\prime} v_{0}=1$.

It follows from the foregoing equation for $J$ that if, for some $r>0$, one has $p(r)=0$, then $u_{2}(s)$ solves equation (1.1) with this $r>0$.

Since we have already proved that $p(R)=0$, the claim follows.

\section{References}

[1] Airapetyan, R., Ramm, A.G., Smirnova, A., Example of two different potentials which have practically the same fixed-energy phase shifts, Phys. Lett A, 254, N3-4, (1999), 141-148. 
[2] Chadan K., Sabatier P., Inverse Problems in Quantum Scattering Theory, Springer, New York, 1989.

[3] J.Cox and K.Thompson, Note on the uniqueness of the solution of an equation of interest in inverse scattering problem, J. Math.Phys., 11, N3, (1970), 815-817.

[4] J.Cox and K.Thompson, On the inverse scattering problem at fixed energy for potentials having nonvanishing first moments, J. Math.Phys., 11, N3, (1970), 805-814.

[5] Newton R., Scattering Theory of Waves and Particles, Springer, New York, 1982.

[6] Ramm, A.G., Recovery of the potential from fixed energy scattering data, Inverse Problems, 4, (1988), 877-886.

[7] Ramm, A.G., Multidimensional inverse scattering problems, Mir Publishers, Moscow, 1994, pp.1-496. (Russian translation of the expanded monograph with the same title published in 1992 by Longman and Wiley).

[8] Ramm, A.G., Property C for ODE and applications to inverse problems, in the book Operator Theory and Its Applications, Amer. Math. Soc., Fields Institute Communications, Providence, RI, vol. 25, (2000), pp.15-75.

[9] Ramm, A.G., Inverse scattering with part of the fixed-energy phase shifts, Comm. Math. Phys., 207, N1, (1999), 231-247.

[10] Ramm, A.G., Analysis of the Newton-Sabatier scheme for inverting fixed-energy phase shifts, Applic. Analysis, 81, N4, (2002), 965-975. 